\title{
Input Admittance of Linear Antennas Driven From a Coaxial Line
}

\author{
Tai Tsun $\mathrm{Wu}^{*}$ \\ Contribution from Gordon McKay Laboratory, Harvard University, Cambridge, Mass
}

(Received April 30, 1962; revised July 30, 1962)

\begin{abstract}
In two cases of a linear antenna driven from a coaxial line, it is shown that the apparent terminal admittance to the coaxial line can be additively separated into two parts when the transverse dimensions are small compared with the wavelength. One of these two parts depends only on the wavelength and the dimensions of the antenna, while the other part can be interpreted as a capacitance that depends only on the radii of the coaxial line. This capacitance may be found exactly from the solution of an integral equation, in the sense that further corrections cannot be interpreted simply as a capacitance.
\end{abstract}

\section{Introduction}

In the experimental determination of the properties of a dipole antenna, the antenna must be driven from a transmission line, usually either a coaxial line or a two-wire line. In particular, the most common method of obtaining the input impedance to the antenna consists of measuring the standing wave ratio and the position of a current minimum on the transmission line. From the point of view of electromagnetic theory, the transmission line and the antenna are inseparable parts of the same problem. However, because of the complexity of the problem, it is often studied, for theoretical purposes, on the basis of an approximation of the following nature. First, the dipole antenna is considered ${ }^{1}$ to be driven by a delta-function generator (sometimes called a slice generator) or perhaps some other generator that is distributed but equally unphysical. From this idealized problem, an input admittance is defined. Then the apparent terminal admittance for the transmission line, i.e., the quantity directly obtainable from the measurements on the transmission line, is found from this input admittance and a lumped corrective network placed at the junction of the transmission line and the dipole antenna [King, 1955]. Such an approximation, is of course, without meaning unless all transverse dimensions of both the transmission line and the dipole antenna are small compared with the wavelength. When these conditions are satisfied and this approximation is taken to be valid, the following statements are usually made:

A. 'The input admittance determined from the idealized antenna problem is independent of the geometry of the transmission line, and

B. The lumped corrective network consists of inductors and capacitors only, and the values of the

\footnotetext{
*Alfred P. Sloan Foundation Fellow. Work also supported in part by Grant G-9721 from the National Science Foundation.

It is not possible to list the relevant references from the vast literature on the dipole antenna. A small sample may be found from footnote 1 of Wu [1961].
}

inductances and the capacitances are independent of the frequency (although they may be negative).

Although this separation into two effects is extremely desirable and is indeed responsible for most of the present understanding of dipole antennas, questions may be raised against both statement $\mathrm{A}$ and statement B. Some of these are the following:

A. For the idealized problem with a delta-function generator, the definition of the input admittance is not entirely straightforward. If it were defined simply as the ratio of the current at the driving point to the driving voltage, then the result would have been infinite. In order to get a finite answer, it is proposed [Wu and King, 1959] that a certain logarithmic singularity in the current distribution should be subtracted out. If $k=2 \pi / \lambda$ is the wave number and $a$ is the radius of the dipole antenna, then this singular term contains a factor $k a$, while the current distribution in general depends inversely on an expansion parameter which in turn depends on the logarithm of the ratio $a / \lambda$. Accordingly, when the antenna is quite thin, this subtraction procedure is probably acceptable. However, it becomes unsatisfactory when the antenna is thicker. For example, when $a / \lambda=0.01, k a$ is about 0.06 while the inverse of the expansion parameter is perhaps slightly more than 0.1. Furthermore, the recent experimental measurements of Rama Rao [private communication] show conclusively that the subtractedout part of the current distribution is actually present on a dipole antenna except possibly very close to the driving point, and hence it cannot be attributed to the idealization of the delta-function generator. Thus, this subtraction procedure deserves further examination. More recent theoretical work [Chen and Keller, 1962; Duncan, 1962] does not substantially improve this situation.

B. Even if the concept of a lumped corrective network is meaningful, it still requires an argument why this lumped network can be represented by frequency-independent inductances and capacitances only. In the case of the dipole antenna driven 
from a two-wire transmission line, the determination of these values involves the introduction of an arbitrary cut-off. And in that case there are reasons to believe that this cut-off ought to depend on the frequency [King, 1956].

Although these questions are present in principle even for the very thin antenna, they are much more relevant from a practical point of view for antennas that are thicker but still satisfy the condition that the transverse dimensions are small compared with the wavelength. Since it now seems very probable that this class of antennas of intermediate thickness will soon be investigated experimentally, it is desirable to have a more critical understanding of these problems. Mathematically, the problem of the antenna with a coaxial transmission line is far simpler than that with a two-wire transmission line because of the absence of complicated corners, and hence, only this simpler case is to be considered. Most of this paper is concerned with the geometry shown in figure 1, namely, an antenna in a parallelplate region driven from a coaxial line. The reasons for choosing this particular geometry are (a) that the corresponding idealized antenna problem with a delta-function generator can be solved exactly by Fourier transform or Fourier series, and (b) that this is a very close approximation to the geometry actually used by Rama Rao in the measurements mentioned above. The same considerations are later applied to the geometry of figure 2, which is much more interesting in connection with possible future measurements on the class of thicker antennas mentioned above.

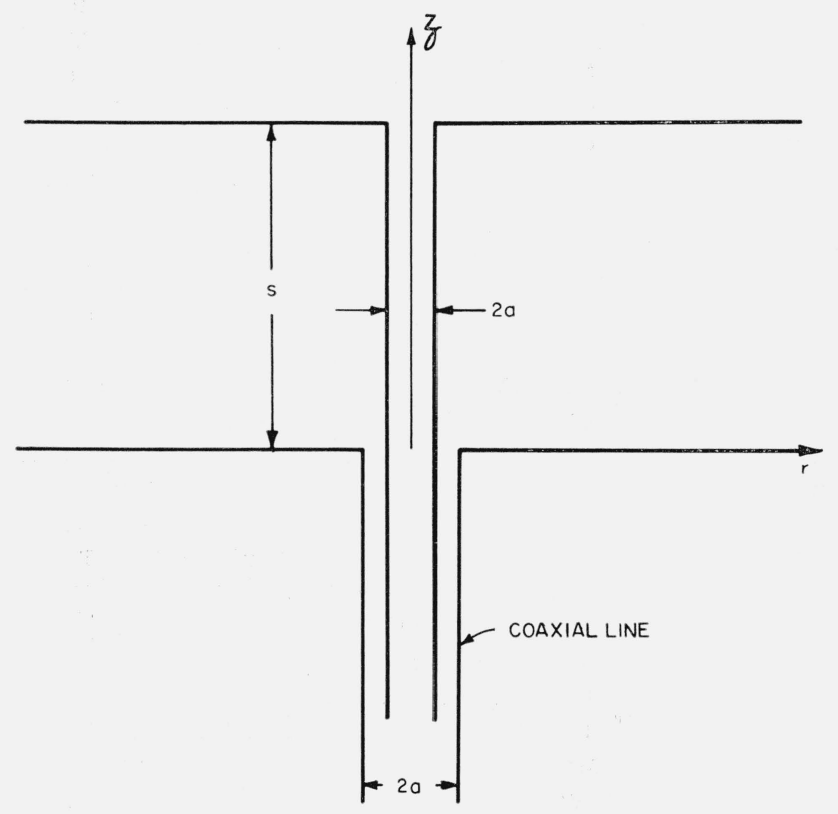

Figure 1. Antenna in a parallel-plate region-driven from a coaxial line.

\section{Idealized Problem With Delta-Function Generator}

In figure 3 is shown the geometry for the idealized problem of an antenna in a parallel-plate region driven by a delta-function generator. Rotational symmetry obtains. Before dealing with the present problem, recall momentarily the situation in the case of a dipole antenna. When a dipole antenna of halflength $h$ is driven at its center by a delta-function generator of voltage $V$, the current distribution on the dipole antenna is determined by the following integral equation [Wu and King, 1959]:

$$
\begin{aligned}
& \int_{0}^{h} d z^{\prime} I\left(z^{\prime}\right)\left[K\left(z-z^{\prime}\right)+K\left(z+z^{\prime}\right)\right] \\
&==4 \pi i \zeta_{0}^{-1} V[\sin k z+2 C \cos k z],
\end{aligned}
$$

where $\zeta_{0}$ is the characteristic impedance of free space,

$$
\begin{aligned}
K(z)=(2 \pi)^{-1} \int_{-\pi}^{\pi} d \theta\left[z^{2}+(2 a \sin \theta / 2)^{2}\right]^{-1 / 2} \\
\quad \times \exp \left\{i k\left[z^{2}+\left(2 a_{*}^{*} \sin _{-}^{-} \theta / 2\right)^{2}\right]^{1 / 2}\right\},
\end{aligned}
$$

and the constant $C$ is determined by the usual boundary condition

$$
I(h)=0 .
$$

By an entirely similar derivation, the current distribution on the antenna for the geometry shown in figure 3 is determined by the following integral equation:

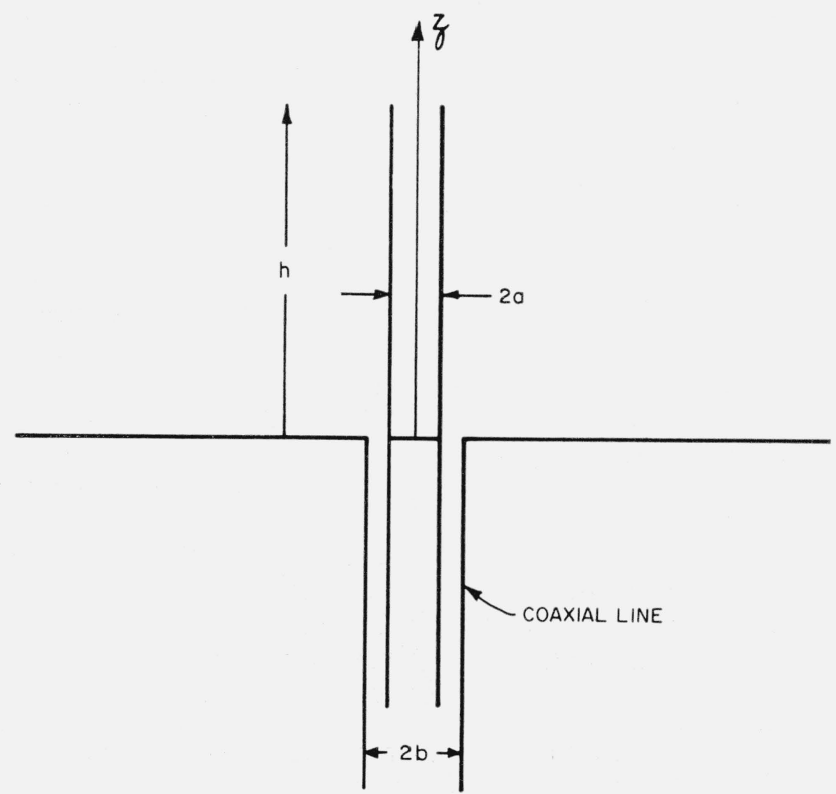

Figure 2. Monopole driven from a coaxial line. 


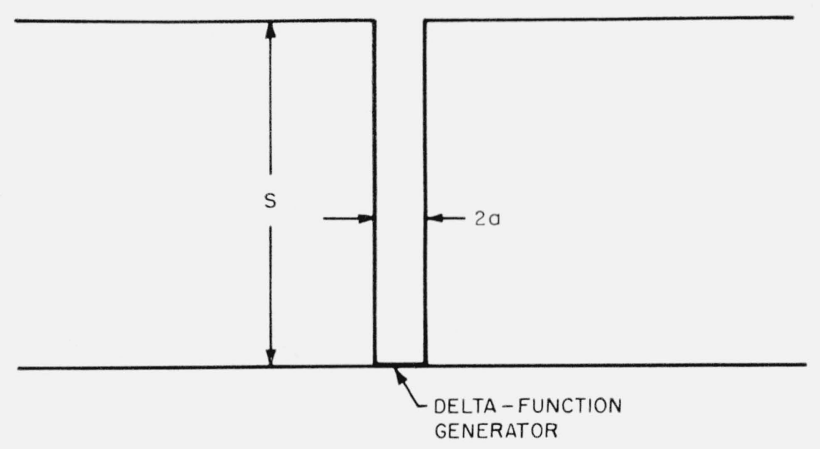

FIGURE 3. Idealized problem with a delta-function generator.

$$
\begin{aligned}
\int_{0}^{s} d z^{\prime} I\left(z^{\prime}\right)\left[K_{p}\left(z-z^{\prime}\right)+K_{p}\left(z+z^{\prime}\right)\right] \\
=4 \pi i \zeta_{0}^{-1} V(\sin k s)^{-1} \cos k(s-z),
\end{aligned}
$$

where $V$ is again the voltage of the delta-function generator, and

$$
K_{p}(z)=\sum_{n=-\infty}^{\infty} K(z+2 n s) .
$$

If $I(z)$ is extended by reflections such that

$$
I(z+2 s)=I(-z)=I(z),
$$

then (2.4) may be rewritten in the alternative form $\int_{-\infty}^{\infty} d z^{\prime} I\left(z^{\prime}\right) K\left(z-z^{\prime}\right)=4 \pi \zeta_{0}^{-1} V \sum_{n=-\infty}^{\infty} \exp [i k|z-2 n s|]$,

where the right-hand side is to be understood in the sense of Abel summability, for example. Equation (2.7) can be solved by Fourier transform with the result

$$
\begin{aligned}
I(z)=- & (4 / \pi) k V \zeta_{0}^{-1} \sum_{n=-\infty}^{\infty} \int_{C_{0}} d \zeta \\
& \times \exp [i \zeta(z-2 n s)]\left(\zeta^{2}-k^{2}\right)^{-1} \\
& \times\left\{J_{0}\left[a\left(k^{2}-\zeta^{2}\right)^{1 / 2}\right] H_{0}^{(1)}\left[a\left(k^{2}-\zeta^{2}\right)^{1 / 2}\right]\right\}^{-1}
\end{aligned}
$$

where the contour $C_{0}$ of integration is shown in figure 4, together with the branch cuts for the integrand. Alternatively, by the Poisson summation formula, (2.8) may be written as

$$
\begin{gathered}
I(z)=4 k V \zeta_{0}^{-1} s^{-1} \sum_{n=-\infty}^{\infty}\left(k^{2}-n^{2} \pi^{2} s^{-2}\right)^{-1} \exp (i n \pi z / s) \\
\left\{J_{0}\left[a\left(k^{2}-n^{2} \pi^{2} s^{-2}\right)^{1 / 2}\right] H_{0}^{(1)}\left[a\left(k^{2}-n^{2} \pi^{2} s^{-2}\right)^{1 / 2}\right]\right\}^{-1} .
\end{gathered}
$$

When $s$ approaches a multiple of $\lambda / 2,\left(k^{2}-n^{2} \pi^{2} s^{-2}\right)$ vanishes, and hence the sum on the right-hand side of (2.9) approaches infinity.

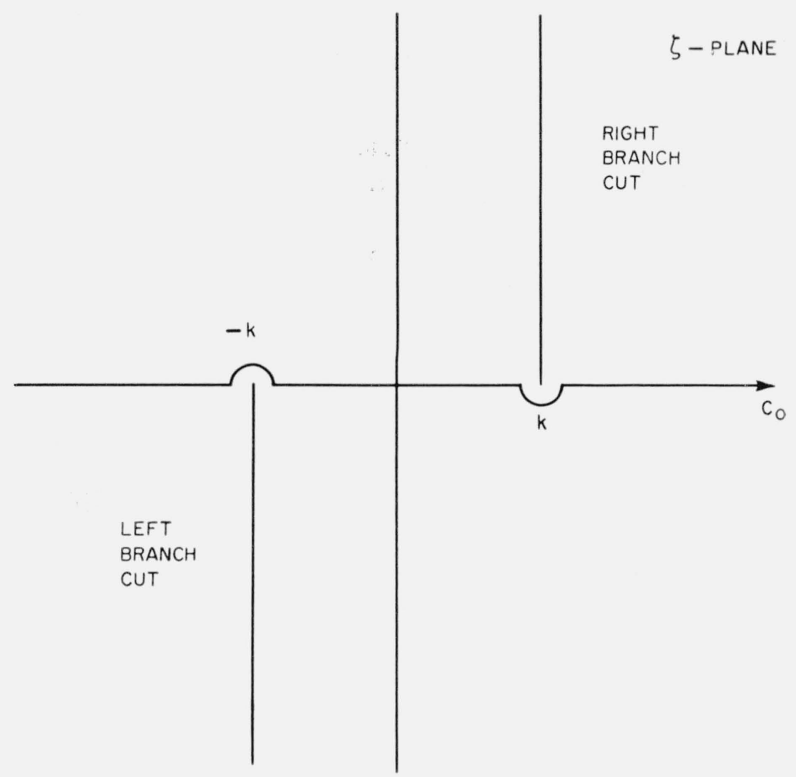

Figure 4. The $\zeta$-plane and the contour $C_{\mathrm{o}}$.

\section{Green's Function for the Parallel-Plate Region}

Let $(r, \theta, z)$ be a cylindrical coordinate system. Let $G_{0}$ be the Green's function in the region ' $r \geq a$ defined by the differential equation

$$
\begin{aligned}
\left(\frac{\partial^{2}}{\partial r^{2}}+\frac{1}{r} \frac{\partial}{\partial r}-\frac{1}{r^{2}}+\frac{\partial^{2}}{\partial z^{2}}+k^{2}\right) G_{0}\left(r, r^{\prime}, z\right) & \\
& =-\frac{1}{r^{\prime}} \delta\left(r-r^{\prime}\right) \delta(z)
\end{aligned}
$$

the Sommerfeld radiation condition, and the boundary condition

$$
(\partial / \partial r) r G_{0}\left(r, r^{\prime}, z\right)=0
$$

for $r=a$. Let $\bar{G}_{0}$ be the Fourier transform of $G_{0}$ defined by

$$
\bar{G}_{0}\left(r, r^{\prime}, \zeta\right)=\int_{-\infty}^{\infty} d z G_{0}\left(r, r^{\prime}, z\right) e^{-i \zeta z},
$$

then, following standard methods given, for example, by Morse and Feshbach [1953], $\bar{G}_{0}$ is explicitly given by

$$
\begin{aligned}
\bar{G}_{0}\left(r, r^{\prime}, \zeta\right)= & \frac{1}{2} i \pi\left[H_{0}^{(1)}(\xi a)\right]^{-1} H_{1}^{(1)}\left(\xi r_{>}\right) \\
& {\left[H_{0}^{(1)}(\xi a) J_{1}\left(\xi r_{<}\right)-J_{0}(\xi a) H_{0}^{(1)}\left(\xi r_{<}\right)\right], }
\end{aligned}
$$

where

$$
\xi=\left(k^{2}-\zeta^{2}\right)^{\frac{1}{2}},
$$

and $r_{>}\left(r_{<}\right)$is the larger (smaller) one of $r$ and $r^{\prime}$. Let 
$\bar{G}_{0}^{(0)}\left(r, r^{\prime}, \zeta\right)=\left(\xi^{2} r r^{\prime}\right)^{-1}\left[\gamma+\ln \frac{1}{2} \xi a-\frac{1}{2} i \pi\right]^{-1}$,

where $\gamma$ is Euler's constant. If all the Bessel functions in (3.4) are expanded in powers of their arguments and only the leading terms retained, the righthand side of (3.6) is obtained. It follows that $\bar{G}_{0}^{(0)}$ is a good approximation of $G_{0}$ when

$$
\left|\xi r_{>}\right| \ll 1
$$

Let $G_{0}^{(0)}$ be the inverse Fourier transform of $\bar{G}_{0}^{(0)}$ then, for $k r>\ll 1, G_{0}^{(0)}$ is a good approximation of $G_{0}$ except when $z$ is small. When $z=0$, the difference is given by

$$
\begin{gathered}
D_{0}\left(r, r^{\prime}\right)=G_{0}\left(r, r^{\prime}, 0\right)-G_{0}^{(0)}\left(r, r^{\prime}, 0\right)=(2 \pi)^{-1} \int_{C_{0}} d \zeta \\
\left\{\frac { 1 } { 2 } i \pi [ H _ { 0 } ^ { ( 1 ) } ( \xi a ) ] ^ { - 1 } H _ { 1 } ^ { ( 1 ) } ( \xi r _ { > } ) \left[H_{0}^{(1)}(\xi a) J_{1}(\xi r<)\right.\right. \\
\left.-J_{0}(\xi a) H_{1}^{(1)}\left(\xi r_{<}\right)\right] \\
\left.\quad-\left(\xi^{2} r r^{\prime}\right)^{-1}\left[\gamma+\ln \frac{1}{2} \xi a-\frac{1}{2} i \pi\right]^{-1}\right\}
\end{gathered}
$$

If $k$ is small, then the right-hand side of (3.8) may be approximated by its value at $k=0$. Hence $D_{0}\left(r, r^{\prime}\right)$ is approximately independent of the wavelength and is given by

$$
\begin{aligned}
& D_{0}\left(r, r^{\prime}\right) \sim \pi^{-1} \int_{0}^{\infty} d \zeta\left\{\begin{array}{c} 
\\
\end{array} K_{0}(\zeta a)\right]^{-1} K_{1}\left(\zeta r_{>}\right)\left[K_{0}(\zeta a) I_{1}\left(\zeta r_{<}\right)\right. \\
&\left.\left.+I_{0}(\zeta a) K_{1}\left(\zeta r_{<}\right)\right]+\left(\zeta^{2} r r^{\prime}\right)^{-1}\left[\gamma+\ln \frac{1}{2} \zeta a\right]^{-1}\right\},
\end{aligned}
$$

where $I_{0}, I_{1}, K_{0}$, and $K_{1}$ are modified Bessel functions.

For the parallel-plate region, define the Green's function $G_{p}$ in the region $r \geq a$ and $|z| \leq s$ by the differential equation

$$
\begin{aligned}
\left(\frac{\partial^{2}}{\partial r^{2}}+\frac{1}{r} \frac{\partial}{\partial r}-\frac{1}{r^{2}}+\frac{\partial^{2}}{\partial z^{2}}+k^{2}\right) & G_{p}\left(r, r^{\prime}, z\right) \\
= & -\frac{1}{r^{\prime}} \delta\left(r-r^{\prime}\right) \delta(z)
\end{aligned}
$$

the Sommerfeld radiation condition, and the boundary condition

$$
(\partial / \partial n) r G_{p}\left(r, r^{\prime}, z\right)=0
$$

when $r=a$ or $z= \pm s$, where $\partial / \partial n$ denotes the normal derivative. It then follows from the method of images that

$$
G_{p}\left(r, r^{\prime}, z\right)=\sum_{n=-\infty}^{\infty} G_{0}\left(r, r^{\prime}, z-2 n s\right) .
$$

In view of (3.6) and (3.12), let

$$
\begin{aligned}
& G_{p}^{(0)}\left(r, r^{\prime}, z\right)=\sum_{n=-\infty}^{\infty} G_{0}^{(0)}\left(r, r^{\prime}, z-2 n s\right) \\
&=\frac{1}{2} s^{-1}\left(r r^{\prime}\right)^{-1} \sum_{n=-\infty}^{\infty}\left(k^{2}-n^{2} \pi^{2} / s^{2}\right)^{-1} \\
& \times \\
& {\left[\gamma+\ln \frac{1}{2}\left(k^{2}-n^{2} \pi^{2} / s^{2}\right)^{\frac{1}{2}} a-\frac{1}{2} i \pi\right]^{-1} e^{i n \pi z / s} . }
\end{aligned}
$$

Then, for $s \gg r_{>}$,

$$
G_{p}\left(r, r^{\prime}, 0\right) \sim G_{p}^{(0)}\left(r, r^{\prime}, 0\right)+D_{0}\left(r, r^{\prime}\right) .
$$

This splits $G_{p}$ into two parts, one part has a very simple dependence on $r$ and $r^{\prime}$, while the other is independent of the wavelength.

\section{Green's Function for the Coaxial Line}

In the region $a \leq r \leq b$, the Green's function for the coaxial line is defined by the differential equation

$$
\begin{aligned}
\left(\frac{\partial^{2}}{\partial r^{2}}+\frac{1}{r} \frac{\partial}{\partial r}-\frac{1}{r^{2}}+\frac{\partial^{2}}{\partial z^{2}}+k^{2}\right) G_{c}\left(r, r^{\prime}, z\right) & \\
= & -\frac{1}{r^{\prime}} \delta\left(r-r^{\prime}\right) \delta(z),
\end{aligned}
$$

the radiation condition for $|z| \rightarrow \infty$, and the boundary conditions

$$
(\partial / \partial r) r G_{c}\left(r, r^{\prime}, z\right)=0
$$

for $r=a$ and for $r=b$. If $\bar{G}_{c}$ is the Fourier transform of $G_{c}$, then it is given by, similar to (3.4),

$$
\begin{aligned}
& \bar{G}_{c}\left(r, r^{\prime}, z\right)=\frac{1}{2} \pi i\left[J_{0}(\xi a) H_{0}^{(1)}(\xi b)-H_{0}^{(1)}(\xi a) J_{0}(\xi b)\right]^{-1} \\
& \times {\left[H_{0}^{(1)}(\xi b) J_{1}\left(\xi r_{>}\right)-J_{0}(\xi b) H_{1}^{(1)}\left(\xi r_{>}\right)\right] } \\
& \times\left[H_{0}^{(1)}(\xi a) J_{1}\left(\xi r_{<}\right)-J_{0}(\xi a) H_{1}^{(1)}\left(\xi r_{<}\right)\right] .
\end{aligned}
$$

When

$$
|\xi b| \ll 1
$$

$\bar{G}_{c}$ is approximated by

$$
\bar{G}_{c}^{(0)}\left(r, r^{\prime}, \zeta\right)=-\left(\xi^{2} r r^{\prime}\right)^{-1}(\ln b / a)^{-1} .
$$

If $G_{c}^{(0)}$ is the inverse Fourier transform of $\bar{G}_{c}^{(0)}$, then again $G_{c}^{(0)}$ is a good approximation, if $k b \ll 1$, to $G_{c}$ except when $z$ is small. Analogous to (3.9), the difference for the special case $z=0$ is given by

$$
\begin{aligned}
& D_{c}\left(r, r^{\prime}\right)=G_{c}\left(r, r^{\prime}, 0\right)-G_{c}^{(0)}\left(r, r^{\prime}, 0\right) \\
& \sim \pi^{-1} \int_{0}^{\infty} d \zeta\left\{-\left[I_{0}(\zeta b) K_{0}(\zeta a)-K_{0}(\zeta a) I_{0}(\zeta b)\right]^{-1}\right. \\
& \times\left[K_{0}(\zeta b) I_{1}(\zeta r>)+I_{0}(\zeta b) K_{1}\left(\zeta r_{>}\right)\right]\left[K_{0}(\zeta a) I_{1}\left(\zeta r_{<}\right)\right. \\
& \left.\left.\quad+I_{0}(\zeta a) K_{1}\left(\zeta r_{<}\right)\right]-\left[\zeta^{2} r r^{\prime} \ln (b / a)\right]^{-1}\right\}
\end{aligned}
$$

More explicitly, 
and

$$
G_{c}^{(0)}\left(r, r^{\prime}, z\right)=i\left[2 k r r^{\prime} \ln (b / a)\right]^{-1} \exp (i k|z|),
$$

$$
G_{c}\left(r, r^{\prime}, 0\right) \sim i\left[2 k r r^{\prime} \ln (b / a)\right]^{-1}+D_{c}\left(r, r^{\prime}\right) .
$$

\section{Antenna in Parallel-Plate Region}

With these properties of the Green's functions in mind, the problem shown in figure 1 can be analyzed. Similar problems have been investigated by, for example, Papadopoulos [1960], but the motivation for the investigation and the method of approach are quite different. Let the incident current on the inner conductor of the coaxial line be

$$
I^{\text {inc }}(z)=e^{i k z} \text {. }
$$

Let $E_{r}(r, z), E_{z}(r, z)$, and $H_{\theta}(r, z)$ be the components of the electromagnetic field corresponding to (5.1). Then $H_{\theta}(r, 0)$ can be expressed in two different ways in terms of $E_{r}(r, 0)$. On the basis of the Green's function for the parallel-plate region, the relation is

$H_{\theta}(r, 0)=-2 i k \zeta_{0}^{-1} \int_{a}^{b} r^{\prime} d r^{\prime} E_{\tau}\left(r^{\prime}, 0\right) G_{p}\left(r, r^{\prime}, 0\right) ;$

if the Green function for the coaxial line is used instead, it is

$$
H_{\theta}(r, 0)=(\pi r)^{-1}-2 i k \zeta_{0}^{-1} \int_{a}^{b} r^{\prime} d r^{\prime} E_{r}\left(r^{\prime}, 0\right) G_{c}\left(r, r^{\prime}, 0\right),
$$

where the extra first term comes directly from (5.1). If the right-hand sides of (5.2) and (5.3) are equated, the following integral equation for $E_{r}(r, 0)$ is obtained:

$$
\begin{aligned}
\int_{a}^{b} r^{\prime} d r^{\prime} E_{r}\left(r^{\prime}, 0\right)\left[G_{p}\left(r, r^{\prime}, 0\right)\right. & \\
& \left.-G_{c}\left(r, r^{\prime}, 0\right)\right]=i(2 \pi r k)^{-1} \zeta_{0} .
\end{aligned}
$$

From here on it is assumed that

$$
k b \ll 1 \text { and } s \gg b ;
$$

hence (3.14) and (4.8) can be used in (5.4).

Let $f(r)$ be the solution of the integral equation

$$
\int_{a}^{b} d r^{\prime} f\left(r^{\prime}\right)\left[D_{0}\left(r, r^{\prime}\right)-D_{c}\left(r, r^{\prime}\right)\right]=r^{-1}
$$

and let

$$
A=\int_{a}^{b} d r f(r) / r
$$

Note that $f(r)$ is independent of $\lambda$, although in general it can be a rather complicated function of $r / a$ and $r / b$. It is desired to express $E_{r}(r, 0)$ in terms of $f(r)$. For this purpose, (3.14) and (4.8) are substituted into (5.4) to give

$$
\begin{aligned}
\int_{a}^{b} r^{\prime} d r^{\prime} & E_{r}\left(r^{\prime}, 0\right)\left[D_{0}\left(r, r^{\prime}\right)-D_{c}\left(r, r^{\prime}\right)\right] \\
& =r^{-1}\left\{i(2 \pi k)^{-1} \zeta_{0}+\tau \int_{a}^{b} d r^{\prime} E_{r}\left(r^{\prime}, 0\right)\right\},
\end{aligned}
$$

where

$$
\begin{array}{r}
\tau=i[2 k \ln (b / a)]^{-1}-\frac{1}{2} s^{-1} \sum_{n=-\infty}^{\infty}\left(k^{2}-n^{2} \pi^{2} / s^{2}\right)^{-1} \\
{\left[\gamma+\ln \frac{1}{2}\left(k^{2}-n^{2} \pi^{2} / s^{2}\right)^{\frac{1}{2}} a-\frac{1}{2} i \pi\right]^{-1} .}
\end{array}
$$

Note that the quantity in the braces of (5.8) is independent of $r$. Accordingly, a comparison of (5.8) with $(5.6)$ shows that $E_{r}(r, 0)$ is given by

$$
E_{r}(r, 0)=\chi f(r) / r
$$

where $\chi$ is independent of $r$ and satisfies

$$
\chi=i \zeta_{0}(2 \pi k)^{-1}+\tau A \chi .
$$

Hence, $\chi$ is explicitly given by

$$
\begin{aligned}
\chi=i \zeta_{0}(2 \pi k)^{-1} & \left\{1-i A[2 k \ln (b / a)]^{-1}\right. \\
& +\frac{1}{2} s^{-1} A \sum_{n=-\infty}^{\infty}\left(k^{2}-n^{2} \pi^{2} / s^{2}\right)^{-1} \\
{[\gamma+} & \left.\left.\ln \frac{1}{2}\left(k^{2}-n^{2} \pi^{2} / s^{2}\right)^{\frac{1}{2}} a-\frac{1}{2} i \pi\right]^{-1}\right\}^{-1} .
\end{aligned}
$$

$\mathrm{By}$ (4.7) the reflected current on the inner conductor of the coaxial line is, for $-z \gg b$,

where

$$
I^{\mathrm{ref}}(z)=\Gamma e^{-i k z},
$$

$$
\Gamma=1-2 \pi \zeta_{0}^{-1}[\ln (b / a)]^{-1} \chi A .
$$

By the theory of transmission lines [King, 1955], the apparent terminal admittance $Y_{a}$ is related to $\Gamma$ by

$$
Y_{a}=Y_{c} \frac{1+\Gamma}{1-\Gamma},
$$

where $Y_{c}$ is the characteristic admittance of the coaxial line. Accordingly, the apparent terminal admittance is given by

$Y_{a}=2 \pi\left[\zeta_{0} \ln (b / a)\right]^{-1}$

$\left\{2-2 \pi\left[\zeta_{0} \ln (b / a)\right]^{-1} \chi A\right\} /\left\{2 \pi\left[\zeta_{0} \ln (b / a)\right]^{-1} \chi A\right\}$.

With (5.12), (5.16) can be expressed in the following form

$$
Y_{a}=Y_{p}-i \omega C_{T}
$$

where

$$
C_{T}=4 \pi \epsilon_{0} A^{-1},
$$

and

$$
\begin{aligned}
Y_{p}=-2 i \pi k \zeta_{0}^{-1} s^{-1} \sum_{n=-\infty}^{\infty}\left(k^{2}-n^{2} \pi^{2} / s^{2}\right)^{-1} \\
{\left[\gamma+\ln \frac{1}{2}\left(k^{2}-n^{2} \pi^{2} / s^{2}\right)^{\frac{1}{2}} a-\frac{1}{2} i \pi\right]^{-1} . }
\end{aligned}
$$


Equation (5.17) gives a separation of the apparent terminal admittance into two parts, one of which is independent of $b$ while the other corresponds simply to an end-correction in the form of a capacitance independent of the frequency and $s$. The value of $C_{T}$ may be found from (5.18), (5.7), (5.6), (3.9), and $(4.6)$.

\section{Current Distribution}

The current distribution on the antenna is given by

$$
I(z)=-4 \pi i k \zeta_{0}^{-1} \int_{a}^{b} r^{\prime} d r^{\prime} E_{r}\left(r^{\prime}, 0\right) G_{p}\left(a, r^{\prime}, z\right) .
$$

Hence, under the assumption (5.5) and for $s \geq z \gg b$, $I(z)$ is given approximately by

$$
\begin{aligned}
I(z)=-2 \pi i k \zeta_{0}^{-1} s^{-1} \chi A \sum_{n=-\infty}^{\infty}\left(k^{2}-n^{2} \pi^{2} / s^{2}\right)^{-1} \\
\\
{\left[\gamma+\ln \frac{1}{2}\left(k^{2}-n^{2} \pi^{2} / s^{2}\right)^{\frac{1}{2}} a-\frac{1}{2} i \pi\right]^{-1} e^{i n \pi / s} . }
\end{aligned}
$$

Comparison with (2.9) shows that the equivalent driving voltage is

$$
V=\chi A \text {. }
$$

Contrary to the remark after (2.9), the right-hand side of (6.2) has no singularity when $s$ is an integral multiple of $\lambda / 2$. Indeed, when $s=m \lambda / 2, \quad(6.2)$ gives

$$
I(z)=2 \cos m \pi z / s .
$$

This has been observed by Rama Rao.

\section{Application to the Dipole Antenna}

With (6.3), (2.9) and (6.2) give the current distribution expressions that are very closely related to each other. This suggests a reinterpretation of the kernel of the integral equation. In this section, statements are made in the context of the geometry of figure 2 , i.e., half of a dipole antenna driven from a coaxial line. These results are perhaps to be expected in view of the conclusions of the last few sections, but they can also be obtained by an analysis of the Green's function for the dipole antenna independent of the similarity to the case of an antenna in a parallel-plate region. Since the principle of the analysis is quite similar to the problem treated above but is somewhat more involved in its details, only the results will be stated here.

Let $h$ be the length of the monopole, and assume that

$$
k b \ll 1 \text { and } h \gg b .
$$

The boundary condition (2.3) applies, but the usual integral equation (2.1) is replaced by

$$
\begin{aligned}
\int_{0}^{h} d z^{\prime} I\left(z^{\prime}\right)\left[\mathscr{K}\left(z-z^{\prime}\right)+\mathscr{K}\left(z+z^{\prime}\right)\right] \\
=4 \pi i \zeta_{0}^{-1} V[\sin k z+2 C \cos k z],
\end{aligned}
$$

where

$$
\mathscr{K}(z)=2[\ln (2 / k a)-\gamma+i \pi / 2] \delta(z)-(d / d z) F(z)
$$

is to be interpreted in the sense of distribution theory [Schwartz, 1950] with $F(z)$ an odd function of $z$ such that

$$
F(z)=-F(-z)=\int_{z}^{\infty} d z^{\prime} e^{i k z^{\prime}} / z^{\prime}
$$

for $z>0$. Since the Fourier transform of $\mathscr{K}(z)$ is

$$
\overline{\mathscr{K}}(\zeta)=2[\ln (2 / k a)-\gamma+i \pi / 2]-\ln \left[\left(k^{2}-\zeta^{2}\right) / k^{2}\right],
$$

the form (7.2) is not new and has effectively been used in connection with the problem of the long dipole antenna [Wu, 1961]. Equation (7.2) leads to a finite $I(0)$, and the input admittance can now be defined simply as

$$
Y=V^{-1} I(0) \text {. }
$$

However, this definition is empty unless $Y$ can be related to $Y_{a}$. This connection is provided by

$$
Y_{a}=Y-i \omega C_{T}
$$

where $C_{T}$ is again determined by (5.18), (5.7), (5.6), (3.9), and (4.6).

The meaning of (7.7) is as follows. The input admittance $Y$ is obtained from the theory outlined in this section, and may be roughly described as the input admittance for a delta-function generator. In this theory, there are only two dimensionless parameters, say $k a$ and $k h$. The feed is highly idealized, and consequently the quantity $Y$ has no direct physical interpretation. On the other hand, in order to carry out an experiment at all, some form of a realistic feed must be used, for example a coaxial line. When such a feed is used, the input admittance of a monopole may be experimentally determined by measurements of the standing wave ratio and the position of a minimum on the feeding line. The value of the input admittance determined in such a manner is the apparent terminal admittance $Y_{a}$. Equation (7.7) states that, if the theoretical input admittance $Y$ is to be compared with the experimental value $Y_{a}$, a term $-i \omega C_{T}$ must be added to $Y$. Note that $C_{T}$ is a function of the radii $a$ and $b$ only, and is independent of $k$ and $h$. Accordingly, this term $-i \omega C_{T}$ is commonly called the capacitive end correction. A more detailed discussion may be found in the book of King [1955].

It may be noted that the Fourier transform of the kernel $\mathscr{K}$ as defined by (7.3) may have a zero on the real axis. In this case, the same contour of integration must be used for inverting the Fourier transform and in the evaluation of the integral of (3.9).

\section{Conclusions and Discussions}

For the case of an antenna driven from a coaxial transmission line, with or without a second ground plane, the apparent admittance terminating the 
coaxial line can be interpreted as the parallel combination of a capacitance $C_{T}$ with an admittance independent of the outer radius of the coaxial line, when (5.5) or (7.1) are satisfied respectively. Except for an overall dimensional factor, the value of $C_{T}$ depends only on the ratio $b / a$. This function of $b / a$, however, has to be computed from the solution of the integral equation (5.6). This procedure gives $C_{T}$ exactly in the sense that further corrections cannot be simply interpreted as a capacitance. The fact that the solution of an integral equation is needed is perhaps not surprising, since the geometry is sufficiently complicated that an integral equation is involved even for the static problem. The admittances independent of the outer radius of the coaxial line, namely $Y_{p}$ and $Y$, are unambiguously specified, explicitly in one case and through a modified integral equation (7.2) in the other case. These specifications are just the same as the one used in connection with the problem of the long antenna [Wu, 1961], but they do not give results in agreement with the subtraction of a logarithmic term proposed earlier [Wu and King, 1959] except for sufficiently thin antennas. The present prescription should be considered to supersede the earlier one [Wu and King, 1959], at least in connection with coaxial transmission lines.

The present work sheds no light on the corresponding problem with a two-wire transmission line. In other words, it remains an open question whether it is proper to consider the apparent admittance terminating a two-wire transmission line as obtainable approximately from the value of $Y$ together with a lumped neiwork realized through the combination of a finite number of frequency-independent inductances and capacitances. This lack of understanding is probably not a serious handicap when the antenna under consideration is very thin. However, so long as this problem remains open, it is almost mandatory to use a coaxial line drive in the experimental measurements of the properties of the class of thicker antennas mentioned in the Introduction.
Finally, it should be mentioned that with slight modifications, the results here can be extended to the case of a lossy medium, i.e., the case of complex $k$. Also, much of the present discussion can be adapted to more general cases involving a coaxial feed; for example, the radii of the antenna and the inner conductor of the coaxial line need not be the same.

For helpful discussions, I am indebted to Professor R. W. P. King, Mr. B. Rama Rao, and Dr. S. R. Seshadri.

\section{References}

Chen, Y. M., and J. B. Keller, Current on and input impedance of a cylindrical antenna, J. Research NBS 66D (Radio Prop.), No. 1, 15-21 (Jan.-Feb. 1962).

Duncan, R. H., Theory of the infinite cylindrical antenna including the feedpoint singularity in antenna current, J. Research NBS 66D (Radio Prop.) No. 2, 181-188 (MarchApril 1962)

King, R. W. P., Transmission-Line Theory (McGraw-Hill Book Company, Inc., New York, 1955).

King, R. W. P., The Theory of Linear Antennas (Harvard University Press, Cambridge, Massachusetts, 1956). See, in particular, pp. 53-54.

Morse, P. M., and H. Feshbach, Methods of Theoretical Physics (McGraw-Hill Book Company, Inc., New York, 1953).

Papadopoulos, V. M., Input admittance of infinitely long dipole antenna driven from coaxial lines, Quart. Appl. Math. 17, No. 4, 423-436 (Jan. 1960).

Schwartz, L., Théorie des distributions, Vol. 1 (Publications de l'Institut de Mathematique de l'Université de Strasbourg, France, 1950).

Wu, T. T., and R. W. P. King, Driving point and input admittance of linear antennas, J. Appl. Phys. 30, No. 1, 74-76 (Jan. 1959).

Wu, T. T., Theory of the dipole antenna and the two-wire transmission line, J. Math. Phys. 2, No. 4, 550-574 (JulyAug. 1961).

(Paper 67D1-246) 